\title{
Review
}

Journal of Innate

Immunity
J Innate Immun 2018;10:3-13

DOI: $10.1159 / 000480373$
Received: May 24, 2017

Accepted after revision: July 28, 2017 Published online: October 7, 2017

\section{Macrophage Extracellular Traps: A Scoping Review}

\author{
Ryan S. Doster ${ }^{a}$ Lisa M. Rogers ${ }^{a} \quad$ Jennifer A. Gaddy ${ }^{a, b}$ David M. Aronoff ${ }^{a}$ \\ ${ }^{a}$ Division of Infectious Diseases, Department of Medicine, Vanderbilt University School of Medicine, and \\ ${ }^{\mathrm{b}}$ Tennessee Valley Healthcare Systems, Department of Veterans Affairs, Nashville, TN, USA
}

\section{Keywords}

Macrophage $\cdot$ Extracellular traps $\cdot$ METosis

\begin{abstract}
Tissue macrophages are derived from either circulating blood monocytes that originate in the bone marrow, or embryonic precursors that establish residence in tissues and are maintained independent of bone marrow progenitors. Macrophages perform diverse functions including tissue repair, the maintenance of homeostasis, and immune regulation. Recent studies have demonstrated that macrophages produce extracellular traps (ETs). ETs are an immune response by which a cell undergoes "ETosis" to release net-like material, with strands composed of cellular DNA that is studded with histones and cellular proteins. ETs are thought to immobilize and kill microorganisms, but also been implicated in disease pathology including aseptic inflammation and autoimmune disease. We conducted a scoping review to define what is known from the existing literature about the ETs produced by monocytes or macrophages. The results suggest that macrophage ETs (METs) are produced in response to various microorganisms and have similar features to neutrophil ETs (NETs), in that METs are produced by a unique cell death program (METosis), which results in release of fibers
\end{abstract}

\section{KARGER}

() 2017 S. Karger AG, Basel

E-Mail karger@karger.com

www.karger.com/jin composed of DNA and studded with cellular proteins. METs function to immobilize and kill some microorganisms, but may also play a role in disease pathology.

(c) 2017 S. Karger AG, Basel

\section{Introduction}

Macrophages comprise a diverse group of cells that are found in all tissues and demonstrate remarkably diverse functions [1-3]. Tissue macrophages are derived from either circulating blood monocytes that originate in the bone marrow, or embryonic precursors that establish residence within tissues and can be maintained independent of bone marrow progenitors $[1,4]$. Macrophage functions range from supporting development, the maintenance of homeostasis, immune surveillance and regulation of tissue remodeling and repair [1]. These professional phagocytes play key roles initiating inflammation and orchestrating its resolution, as seen by the spectrum of macrophage "activation states" [3]. In addition to classic macrophage functions, recent investigation has dem-

J.A.G. and D.M.A. contributed equally to this work.
Dr. David M. Aronoff

Division of Infectious Diseases, Vanderbilt University School of Medicine A2200 Medical Center North, 116121 st Avenue South

Nashville, TN 37232 (USA)

E-Mail d.aronoff@vanderbilt.edu 
Fig. 1. Search and review flowchart: 244 articles were identified by the primary PubMed search, 3 were excluded based on language, 241 underwent initial review of the abstracts, and 229 were excluded. Ultimately, 12 primary articles were identified for inclusion in this review based on the primary search; from the references of these articles, an additional 9 articles were identified. ETs, extracellular traps; NETs, neutrophil extracellular traps; METs, macrophage extracellular traps.

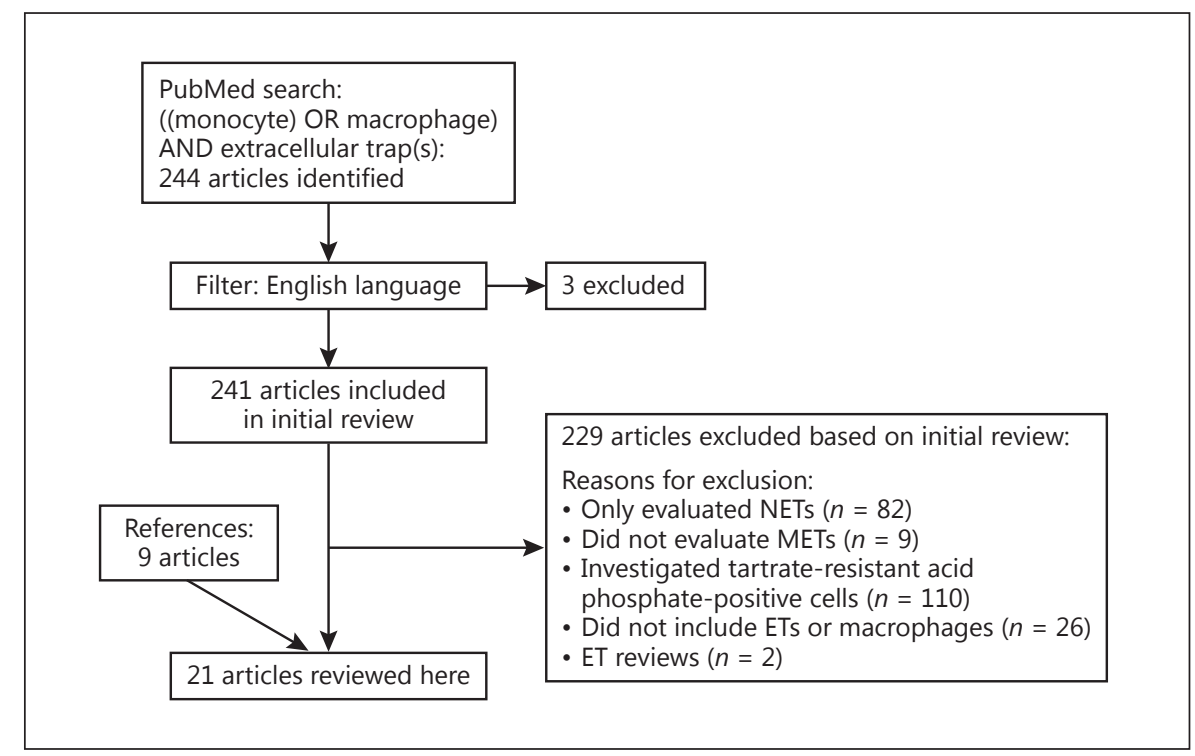

onstrated that macrophages are capable of producing extracellular traps (ETs).

An initial description of neutrophil extracellular traps (NETs) appeared in 2004, and this discovery was quickly followed by description of a new, programmed cell death pathway termed "ETosis" [5-7]. ETosis comprises a unique series of cellular events by which nuclear contents, including chromatin, mix with cellular proteins and are then extruded from the cell body to form extracellular structures capable of "trapping" and killing microorganisms [5-7]. Since the original report about neutrophils, other leukocytes including mast cells, eosinophils, and basophils are now known to produce "extracellular trap" structures [8-12]. ETs have been implicated in diverse disease states ranging from conditions of aseptic inflammation, such as gout, to vascular disorders including preeclampsia and thrombosis [13-16]. Inadequate resolution and degradation of these structures is also a topic of recent research; prolonged exposure of self-antigens comprising ETs can result in autoimmune diseases including systemic lupus erythematosus (SLE) and antineutrophil cytoplasmic autoantibodies (ANCA)-associated vasculitis $[17,18]$.

As macrophage functions are vital to host immune defense and tissue homeostasis, we sought to understand the role of macrophage and monocyte ETs (METs) in the context of host defense and disease states. We conducted a structured, scoping review to assess the current literature regarding the diversity of macrophages known to produce ETs, the identification of METs, the proposed functions, and the mechanisms by which macrophage ETosis
(METosis) may occur. Data regarding ETs produced by neutrophils and other leukocytes have been reviewed elsewhere and were excluded from this review $[19,20]$.

\section{Methods}

We performed a scoping review based on methods described by Arksey and O'Malley [21]. The central research question was: "What is known from the existing literature about ETs produced by monocytes or macrophages?" To address this, we searched for and reviewed papers with the following inclusion criterion: English language primary studies that provided some evaluation of monocytes or tissue differentiated macrophages for the ability to produce ETs. For the purpose of this review, we refer to both monocyte and macrophage ETs as "METs" and the process of extruding these structures as "METosis." These studies were not limited to human cells.

Our search was conducted using PubMed, with the search phrase: monocyte OR macrophage AND extracellular traps(s). The last search for new manuscripts was performed on 30 March 2017. The search is summarized in Figure 1. We identified additional studies via the references of the reviewed articles. Further review to include/exclude identified articles consisted of reading the abstracts. If an article appeared relevant, but no inclusion criteria could be established, the full article was reviewed to determine if it satisfied the inclusion criterion.

\section{Results}

\section{Defining and Identifying METs}

The original description of NETs included high-resolution scanning electron microscopy (SEM) imaging; 
Fig. 2. Examples of METs. a Scanning electron micrograph image of a placental macrophage with expelled MET in response to $S$. agalactiae. Scale bar, $20 \mu \mathrm{m}$. As can be seen in the magnified panels (right), these fibers originate from the macrophage cell (bottom), and bacterial cells become embedded in these fibers (top). b Placental macrophage infected with $S$. agalactiae and treated with DNase I, leaving a defect in the macrophage cell surface where MET fibers exited the cell. Scale bar, $5 \mu \mathrm{m}$. c Confocal microscopy $3 \mathrm{D}$ reconstruction image of a placental macrophage MET in response to $S$. agalactiae. Scale bar, $10 \mu \mathrm{m}$. Cells were stained with Sytox green (green) for extracellular DNA and Hoechst 33342 (blue) for condensed chromatin. Extracellular DNA fibers (green) are seen extending beyond the nucleus structure (blue). Placental macrophages were obtained as published from deidentified, term, nonlabored placentae provided by the Cooperative Human Tissue Network, which is funded by the National Cancer Institute [59]. All tissues were collected in accordance with Vanderbilt University Institutional Review Board approval (\#131607). d, e PMAdifferentiated THP-1 cells, an immortalized a monocyte cell line, produce METs (white arrows) in response to $S$. agalactiae, and these structures are absent when treated with DNase I (e). Cells were stained with Sytox green (green) and Hoechst 33342 (blue). Scale bars, $40 \mu \mathrm{m}$.

with this modality, Brinkmann et al. [5] identified fibers that were $15-17 \mathrm{~nm}$ in diameter and beaded with globular domains. Using immunofluorescence staining, this study determined that these globular domains contained proteins from neutrophil azurophilic, secondary, and tertiary granules including elastase, myeloperoxidase (MPO), lactoferrin, and gelatinase. Interestingly, these extracellular fibers did not contain cytoskeleton components or other cytoplasmic proteins. DNA was found to be the major structural component of these fibers, demonstrated by staining with DNA intercalating dyes and the destruction of these structures when treated with DNase.

The time course by which cells undergo ETosis seems to be variable. The original description of NETs demon-
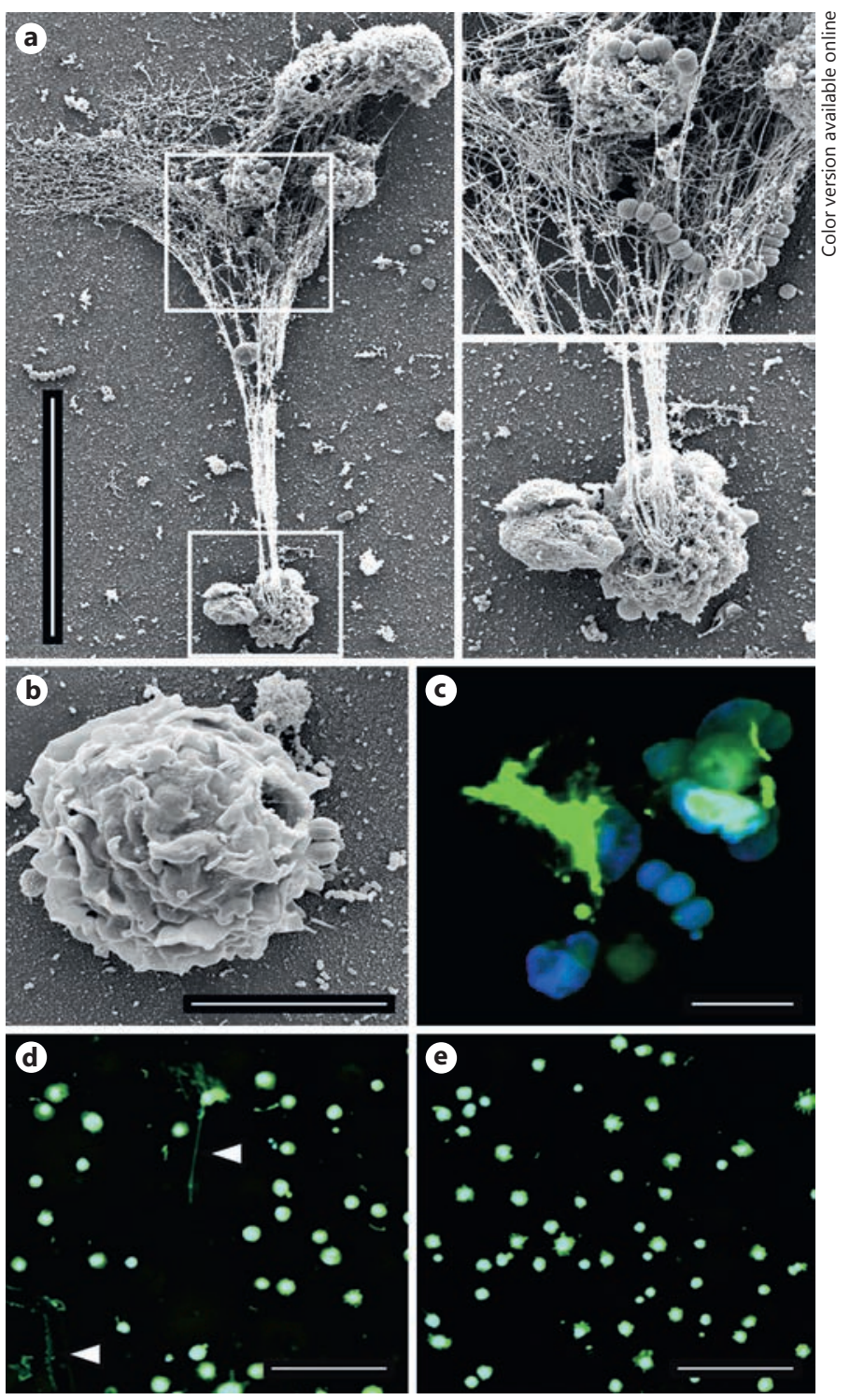

strated rapid release of extracellular DNA that occurred in as little as $10 \mathrm{~min}$ following stimulation with phorbol myristate acetate (PMA), but later reports have suggested ETosis could take several hours [5, 6]. Yousefi et al. [9] described DNA release from eosinophils within $5 \mathrm{~min}$ of stimulation with complement component $\mathrm{C} 5 \mathrm{a}$ or lipopolysaccharide (LPS), and the maximum effect, measured as fluorescence of a cell-impermeable DNA-staining dye, occurred within $30 \mathrm{~min}$. METosis has been described as a rapid process that can occur in $<30 \mathrm{~min}[22,23]$.

In order to identify METs, most studies utilize either SEM, looking for fibers similar to those described by Brinkmann et al. [5], or fluorescent DNA-binding dyes, such as Sytox ${ }^{\circledR}$, PicoGreen ${ }^{\circledR}$, TO-PRO ${ }^{\circledR}-3$, DAPI, or 
Table 1. Types of monocytes and macrophages known to produce METs and the cellular proteins identified within MET structures

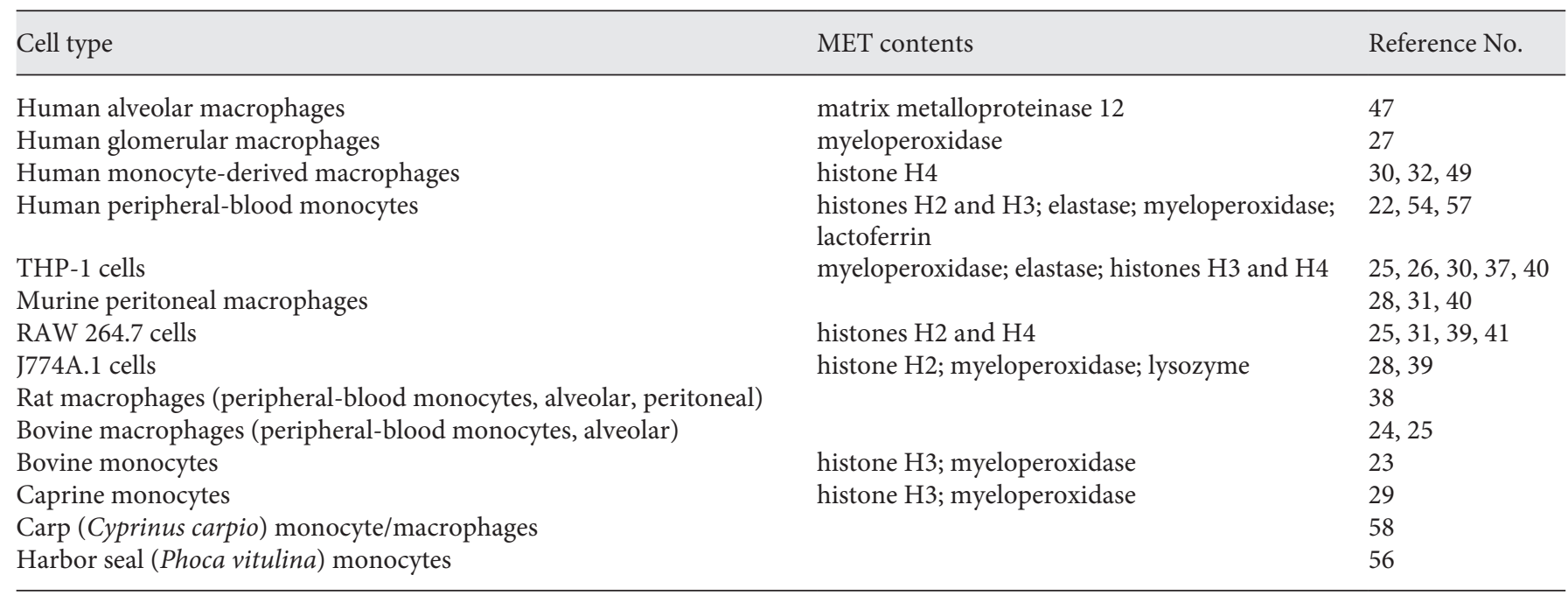

Hoechst, visualized by scanning laser confocal microscopy. These dyes have also been used to construct spectofluorometric assays as an effort to quantify MET release by measuring fluorescence staining of extracellular DNA $[6,24,25]$. Typical definitions of METs, like NETs, include extracellular fibers composed of DNA that extend outside the bounds of the cell and are degraded by treatment with DNase I or micrococcal nuclease (Fig. 2). Additional verification that proposed structures are ETs can be obtained by staining for known ET components such as histones, elastase, or MPO. Elastase, often considered a neutrophil-specific marker, has been identified in the METs of human peripheral-blood monocytes and in THP-1 macrophage-like cells [22, 26]. Similarly, MPO has been identified in the METs of diverse macrophage populations including human glomerular macrophages, human peripheral-blood monocytes, THP-1 macrophage-like cells, murine J774A.1 macrophage-like cells, bovine monocytes, and caprine monocytes [22, 23, 2629] (Table 1).

\section{Factors That Influence MET Release}

As is the case with NETs, diverse pathogens and chemical stimuli have been noted to induce METs (Table 2). In addition to live bacterial cells, Wong and Jacobs [30] described that particular virulence factors such as the $M y$ cobacterium tuberculosis secretion system, ESX-1, may specifically contribute to MET release by human peripheral-blood monocyte-derived macrophages, a result that was augmented in the presence of other chemical activators including interferon- $\gamma$. Aulik et al. [25] noted that
Mannheimia haemolytica infection of bovine monocytederived macrophages led to MET release in a leukotoxindependent manner as infection with leukotoxin-deficient M. haemolytica cells did not result in MET release. Several publications have noted that the proinflammatory mediators that stimulate generation of reactive oxygen species (ROS) induce METs, though this finding is not consistent across all cell types and experimental conditions [25, 31]. Liu et al. [28] demonstrated that treatment of mouse macrophage-like J774A.1 cells or primary mouse peritoneal macrophages with PMA, hydrogen peroxide, interferon- $\gamma(100 \mathrm{U} / \mathrm{mL})$, or macrophage colony-stimulating factor $(25 \mathrm{ng} / \mathrm{mL})$ did not result in MET release, but that infection with Candida albicans or Escherichia coli was able to stimulate METosis. Based on this evidence, the authors suggested that the extrusion of MET structures from these cells occurred by a ROS-independent mechanism.

Despite the great diversity of tissue macrophages, few studies currently exist regarding the different types of macrophages that respond to pathogens with MET release (Table 1). Knowledge is also limited regarding why some macrophages undergo METosis while others do not. For instance, Bonne-Année et al. [32] noted that although human monocyte-derived macrophages produced METs in response to Strongyloides stercoralis infection, mouse peritoneal macrophages did not, despite the fact that both human and mouse neutrophils produced NETs during infection. Similarly, Schorn et al. [10] reported that neutrophils, basophils, and eosinophils responded to monosodium urate crystals in gouty arthritis 
Table 2. Microbial organisms that elicit a MET response and chemical inducers and inhibitors of METosis

\begin{tabular}{|c|c|c|c|c|c|c|}
\hline $\begin{array}{l}\text { Gram-negative } \\
\text { bacteria [Ref.] }\end{array}$ & $\begin{array}{l}\text { Gram-positive } \\
\text { bacteria [Ref.] }\end{array}$ & $\begin{array}{l}\text { Parasites } \\
\text { [Ref.] }\end{array}$ & $\begin{array}{l}\text { Acid-fast bacilli } \\
\text { [Ref.] }\end{array}$ & $\begin{array}{l}\text { Fungi } \\
\text { [Ref.] }\end{array}$ & $\begin{array}{l}\text { Chemical inducers } \\
\text { [Ref.] }\end{array}$ & $\begin{array}{l}\text { Chemical inhibitors } \\
\text { [Ref.] }\end{array}$ \\
\hline $\begin{array}{l}\text { Escherichia coli } \\
{[22,28,54]} \\
\text { Nontypeable } \\
\text { Haemophilus } \\
\text { influenza } \\
{[47]} \\
\text { Histophilus } \\
\text { somni } \\
{[24]} \\
\text { Klebsiella } \\
\text { pneumoniae } \\
{[54]} \\
\text { Mannheimia } \\
\text { haemolytica } \\
{[25]}\end{array}$ & $\begin{array}{l}\text { Staphylococcus } \\
\text { aureus } \\
{[22,31,40]} \\
\text { Streptococcus } \\
\text { agalactiae } \\
{[39]}\end{array}$ & $\begin{array}{l}\text { Besnoitia besnotii } \\
{[23]} \\
\text { Eimeria ninakohlyakimovae } \\
{[29]} \\
\text { Strongyloides stercoalis } \\
{[32]} \\
\text { Toxoplasma gondii } \\
{[56]}\end{array}$ & $\begin{array}{l}\text { Mycobacterium } \\
\text { abscessus } \\
{[57]} \\
\text { M. massiliense } \\
{[26]} \\
\text { M. tuberculosis } \\
{[30]}\end{array}$ & $\begin{array}{l}\text { Candida } \\
\text { albicans } \\
{[22,28]}\end{array}$ & $\begin{array}{l}\text { fosfomycin } \\
{[40]} \\
\text { glucose oxidase } \\
{[25]} \\
\text { HMG-CoA reductase } \\
\text { inhibitors (statins) } \\
{[31]} \\
\text { interferon- } \gamma \\
{[30]} \\
\text { NET material } \\
{[37]} \\
\text { phorbol myristate acetate } \\
{[25,31]} \\
\text { TNF- } \alpha \\
{[41]} \\
\text { zymosan } \\
{[23,56]}\end{array}$ & $\begin{array}{l}\text { 4-aminobenzoic acid } \\
\text { hydrazide (ABAH) } \\
{[23]} \\
\text { apocinin } \\
{[47]} \\
\text { cytochalasin D } \\
{[25,26]} \\
\text { diphenylene iodonium } \\
{[23,25,29,40]} \\
\text { GM-CSF } \\
{[30]} \\
\text { N-methoxysuccinyl-Ala-Ala- } \\
\text { Pro-Val-chloromethyl } \\
\text { ketone } \\
{[26,30,40]} \\
\text { z-YVAD-fmk } \\
{[54]}\end{array}$ \\
\hline
\end{tabular}

by producing ETs, but peripheral-blood monocytes, despite phagocytosing the crystals, did not produce METs. Chow et al. [31] made the interesting observation that treating either mouse RAW 264.7 macrophage-like cells or primary mouse peritoneal macrophages with members of the statin family of cholesterol-lowering drugs resulted in enhanced MET release from these cells, but Halder et al. [22] were not able to demonstrate similar responses with human peripheral-blood monocytes. Current evidence is insufficient to determine if the differential responses are due to experimental conditions or if some types of macrophages are inherently more prone to METosis.

Aside from the interactions between macrophages and specific microorganisms, other factors including the cellular environment and polarization state may alter a macrophage's ability to undergo METosis. As for macrophages, the neutrophil literature reveals a growing appreciation of distinct neutrophil subsets that play particular roles in immunity and disease pathogenesis. These subsets, including proinflammatory, antitumorigenic N1 and tumorigenic N2, represent spectrums of cellular phenotypes that differ in their morphological and functional properties [33]. Type I interferons have recently been shown to promote a proinflammatory subset of neutrophils (N1, antitumorigenic) which display an enhanced ability to form NETs [34]. Likewise, Shrestha et al. [35] used retinoic acid to drive neutrophil nuclear hypersegmentation, a characteristic finding of N1 cell morphology, and found that retinoic acid treatment also resulted in the augmented release of NETs in response to PMA stimulation. In both studies, the NET-producing, N1 neutro- phils also exhibited an increased capacity for cytotoxicity against cancer cells. Shrestha et al. [35] demonstrated that the addition of DNase reversed this effect, suggesting an antitumor role for NETs. Additionally, Villanueva et al. [36] demonstrated that a subset of "low-density granulocytes" isolated from patients with SLE had a greater capacity to produce NETs than normal-density neutrophils from patients with SLE or control neutrophils from patients without SLE. By producing a higher proportion of NETs, these cells may provide increased exposure of autoantigens and have an increased capacity to kill endothelial cells, driving disease pathogenesis.

Macrophages are known to function on a spectrum of activated phenotypes, or, polarization states [3]. Only 1 report to date specifically investigated how macrophage polarization may influence METosis. Nakazawa et al. [37] made the observation that PMA differentiated THP-1 cells polarized toward an inflammatory M1 activation state (treated with $20 \mathrm{ng} / \mathrm{mL}$ IFN- $\gamma$ and $1 \mathrm{mg} / \mathrm{mL}$ LPS for $6 \mathrm{~h}$ ), but not the anti-inflammatory/prohealing M2 state (treated with $20 \mathrm{ng} / \mathrm{mL} \mathrm{IL}-4$ for $24 \mathrm{~h}$ ), resulted in extracellular DNA release when exposed to NET material. Of note, the authors did not specifically label this extracellular DNA release as METs, but their findings suggest that this extracellular DNA extended beyond cellular boundaries and originated from the macrophages, despite noting that the DNA release was "less drastic" than that seen by neutrophils undergoing ETosis. They found similar results with peripheral-blood mononuclear cells differentiated to polarized macrophages after 7 days of incubation with $10 \mathrm{ng} / \mathrm{mL}$ of GM-CSF (M1) or M-CSF (M2). Interestingly, DNA release by THP-1 macrophage-like cells 
was specific to the interaction with NETs, as macrophage extracellular DNA release was not stimulated by interactions with neutrophils undergoing other types of cell death.

Changes in the cellular environment might also alter macrophage behavior. Bryukhin and Shopova [38] used a rat model of liver injury, which has been shown to depress immune function including monocyte migration and phagocytic activity, to demonstrate that alveolar macrophages and peripheral-blood monocytes taken from the pups of mothers with drug-induced hepatitis were impaired in MET formation and bactericidal activity. Their work suggests that cellular programming or environmental signals may modify the ability of macrophage to complete METosis. Vega et al. [39] demonstrated that mouse J774A.1 and RAW264.7 macrophage-like cells undergoing a stress response (incubation at $42^{\circ} \mathrm{C}$ for $1.5 \mathrm{~h}$ followed by recovery at $37^{\circ} \mathrm{C}$ ) were more prone to produce METs when exposed to Streptococcus agalactiae. In their experimental conditions, S. agalactiae infection resulted in decreased ROS/RNS production and elevated levels of IL-10, which are most typically associated with ET inhibition in neutrophils. They suggested that changes in the cytoskeleton triggered by stress responses, potentially via Hsp70 and Hsp27, might lead to METosis in the absence of oxidative stress or proinflammatory mediators.

Together, these studies demonstrate that MET release can occur in response to a diverse collection of pathogens, but data are limited regarding the responses of specific types of tissue macrophages. More work is needed to better understand how polarization states and environmental signals might alter cellular function with respect to METs, and whether these changes would affect all tissue macrophages in a similar way. Future studies will be important as the functions of METs in both immunity and pathophysiology become clearer.

\section{Functions Attributed to METs}

Several functions have been attributed to NETs including trapping diverse pathogens, suppression and destruction of the bacterial toxins of trapped organisms, and bactericidal activity [5]. The bactericidal activity of NETs has been attributed to a localized, high concentration of antimicrobial peptides contained on the globules of NET fibers as DNase treatment eliminates this cidal activity [5]. Several studies have examined the fundamental question of whether METs have cidal activity for different microorganisms. In order to assess microbicidal activity, most MET killing assays compare macrophages infected with or without the presence of DNase, and compare the recovered microorganisms $[24,25]$. In order to separate killing that results from MET-dependent and MET-independent mechanisms (mainly phagocytosis), some studies have used the actin cytoskeletal inhibitor, cytochalasin $\mathrm{D}$, to inhibit macrophage phagocytosis [24, 28]. There have been mixed results using this technique as some groups have noted decreased MET release in cells pretreated with cytochalasin $\mathrm{D}[25,26]$. To date, there are reports of potential MET microbicidal activity against pathogens including Staphylococcus aureus, S. agalactiae, E. coli, and C. albicans [28, 38-40]. Halder et al. [22] isolated monocyte ETs by digesting immobilized traps with the restriction enzyme $A l u \mathrm{I}$, and then added ET material to C. albicans culture; ET material, but not monocyte chromosomal DNA, was able to inhibit fungal growth even at $30 \mathrm{~h}$ of incubation. Instead of employing direct counting of microorganisms, Chow et al. [31] used SYTO $^{\text {TM }} 9$ and propidium iodide staining of $S$. aureus cells trapped within METs to demonstrate the decreased viability of bacterial cells.

In spite of the results stated above, several studies have questioned the direct bactericidal activity of METs. For example, a recent study evaluated infection of PMA-differentiated THP-1 macrophage-like cells with Mycobacteria massiliense and noted that MET release actually enhanced bacterial growth, potentially by providing a scaffold for bacterial aggregation, which facilitates survival in this organism [25]. Liu et al. [28] demonstrated that mouse J774A.1 macrophage-like cells infected with E. coli or C. albicans resulted in small but significant microbial killing attributed to METs (10-20\% less organisms recovered than in cells treated with DNase and cytochalasin D), but peritoneal macrophages in similar assays did not show any significant MET microbicidal activity against either organism. Of note, in this study, the extent of microbial killing attributed to METs was considerably less than that attributed to intracellular killing.

It has been proposed that METs may act synergistically with other components of host defense. Halder et al. [22] demonstrated that human monocyte release of METs was enhanced in the presence of human serum. During infection with $C$. albicans in media containing serum, they used immunofluorescence staining to establish that complement factors C $3 \mathrm{~b}$ and C5b-9 were deposited onto METs. They proposed that activated complement might add microbicidal activity and allow for the enhanced opsonization and phagocytosis of organisms within ETs during the resolution of inflammatory responses [22]. Shen et al. [40] showed that the antibiotic fosfomycin may 
boost MET release, potentially by increasing ROS production, and enhance the total extracellular killing of $S$. aureus by mouse peritoneal macrophages producing METs.

One of the difficulties of these studies is successfully demonstrating that METosis and METs occur in vivo and are not an artifact of ex vivo experimental conditions. Just as NETs are now recognized in diverse tissues, METs have been identified within tissues during disease states. O'Sullivan et al. [27] evaluated renal biopsy tissues from patients with MPO-ANCA-associated glomerulonephritis. Tissues from patients with glomerulonephritis had significantly more macrophages than control tissues. The authors used immunofluorescent probes against nuclear components and MPO to identify NETs and METs, which they differentiated by the presence of neutrophil elastase (a NET marker) and CD68 positivity (a MET marker). Six of 10 biopsies from patients with glomerulonephritis were found to have MPO-containing METs. This is particularly interesting given that MPO, a component of both NETs and METs, is considered to be a major autoantigen in the development of ANCA-associated vasculitis, suggesting that macrophage and neutrophil ETs contribute to the pathophysiology of this disease. Mohanan et al. [41] examined macrophage function at "crown-like structures" in breast adipose tissue. Crownlike structures are thought to be areas where macrophages clear dying adipocytes. This study evaluated mammary adipose tissue from obese mice, and found that macrophages at crown-like structures stained strongly for $\mathrm{PAD}_{2}$ and citrullinated histones (anti-H4Cit3) which extended from macrophage nuclei into the extracellular space. The authors proposed that these macrophages were undergoing hypercitrullination via $\mathrm{PAD}_{2}$ as part of METosis. This report suggested that METosis in adipose tissue could be driven by TNF- $\alpha$ release from dying adipocytes. Proinflammatory signals may promote MET release and drive further inflammatory signals within adipose tissue, influencing macrophage infiltration and activation.

\section{Cellular Pathways Implicated in MET Release}

Identifying mechanisms by which neutrophils undergo ETosis, and subsequently release NETs, continues to be an area of active research. The "NETotic cascade" comprises several steps including cytoplasmic and nuclear swelling, vacuolization, membrane protrusion, enzyme-binding to DNA, histone citrullination and chromatin decondensation, terminating in membrane rupture and NET release [42]. During this cascade, cellular granule membranes also break down, allowing for nucle- ar and granular proteins to mix and for granular proteins like elastase to be found within the NETs after release [6]. Early descriptions of NETs noted that this process was dependent on the NADPH oxidase system, in which NADPH oxidase-derived ROS acted intracellularly to initiate the NET cascade via actions that include the activation of elastase, which then escapes from neutrophil azurophilic granules $[42,43]$. Once released, elastase translocates to the nucleus, where it degrades histones and thereby promotes chromatin decondensation [43]. Reports have also suggested that interactions between ROS formation, cellular autophagy, and $\mathrm{PAD}_{4}$-dependent histone citrullination result in the collapse of intracellular membranes, allowing for chromatin decondensation and subsequent release [44].

Alternative forms of ETosis with unique pathways are now being described. Yousefi et al. [45] reported that neutrophils primed with GM-CSF and stimulated with LPS or complement component C5a could produce NETs from mitochondrial DNA in a pathway that depended on ROS but did not result in cellular death. Pilscek et al. [46] described another alternative form of neutrophil ETosis in which nuclear DNA was deposited extracellularly via membrane vesicles, a process which was not inhibited by diphenylene iodonium, suggesting an NADPH-independent pathway.

Investigations into mechanisms of MET release have been less numerous. Reports have demonstrated that inhibitors of the NADPH-oxidase system including diphenylene iodonium reduce MET formation, although this has not been consistent across all studies [23, 25, 28, 29, 40]. King et al. [47] used a fluorescent readout of ROS production (dihydrorhodamine 123) to indicate that human alveolar macrophages producing METs had a 2-fold increase in ROS fluorescence compared to cells not forming METs. Treating alveolar macrophages with the ROS inhibitor apocinin inhibited MET release. Aulik et al. [25] established that other pathways boost intracellular ROS including treatment with glucose oxidase or PMA enhanced MET release from bovine macrophages.

As with neutrophils, there are reports of alternative pathways of METosis [26, 28]. Je et al. [26] suggested that calcium influx into PMA-differentiated THP-1 macrophage-like cells may influence MET production by means of an NADPH oxidase-independent mechanism. Treatment of THP-1 cells with $20 \mu \mathrm{M}$ 1,2-bis(o-aminophenoxy)ethane- $\mathrm{N}, \mathrm{N}, \mathrm{N}^{\prime}, \mathrm{N}^{\prime}$-tetraacetic acid or $1 \mu \mathrm{M}$ ethylene glycol tetraacetic acid to chelate calcium diminished, but did not eliminate, MET release. Interestingly, this report suggested that the DNA present in these METs was com- 
posed of nuclear and mitochondrial DNA as the authors were able to identify nuclear ( $A C T B, G A P D H)$ and mitochondrial (ATP6, NDS1) genes in the supernatants of infected cells by PCR. Similarly, Liu et al. [28], who also proposed an ROS-independent mechanism of MET release from mouse J774A.1 macrophage-like cells, identified both mitochondrial (Atp6 and Nds1) and nuclear ( $A c t b$ and Gapdh) genes via in situ hybridization and PCR amplification of the supernatants from $C$. albicans-stimulated macrophages.

Other important steps in METosis remain poorly understood. In neutrophils, both elastase and MPO contribute to ETosis $[43,48]$. Elastase promotes NET release by degrading histones and promoting chromatin decondensation, and MPO synergizes with elastase chromatin decondensation in a mechanism that is independent of enzymatic activity [43]. To date, 4 studies have evaluated if elastase and myeloperoxidase contribute to METosis. Wong and Jacobs [30] used the elastase inhibitor, $\mathrm{N}$ methoxysuccinyl-Ala-Ala-Pro-Val-chloromethyl ketone (AAPV), to block monocyte-derived macrophages infected with $M$. tuberculosis or S. aureus from releasing METs. Shen et al. [40] found similar results with AAPVtreated mouse peritoneal macrophages infected with $S$. aureus. In contrast, Je et al. [26] noted AAPV-treated THP-1 macrophage-like cells infected with M. massiliense were unaffected with regard to MET release despite elastase being identified within the MET structures. Muñoz-Caro et al. [23] inhibited MET release by bovine monocytes infected with Besnoitia besnotii trachyzoites using the MPO inhibitor, 4-aminobenzoic acid hydrazide (ABAH). These studies all suggest an active role for elastase and MPO in METosis, but it remains to be determined if the mechanisms by which these enzymes contribute actually mirror those seen in neutrophils.

Chow et al. [31] used statin drugs to implicate sterol production pathways as a potentially important mediator of MET formation. They demonstrated that MET formation was enhanced in statin-treated murine peritoneal macrophages. They found similar results using siRNA knockdown of HMG-CoA reductase; this was reversed by treatment with the HMG-CoA reductase product mevalonate. They proposed that the effect of statins to boost MET production might be mediated by intermediates of the sterol synthetic pathways, although the exact mechanism by which these intermediates may alter METosis is not well understood.

The role of cytoskeleton rearrangement in both neutrophils and macrophage ETs remains a topic of debate. Pretreatment with the actin cytoskeletal inhibitor, cyto- chalasin D, often used to block phagocytosis, has demonstrated the ability to decrease MET formation in some studies $[25,26]$. Cytochalasin D treatment did not have a significant effect on METosis of bovine monocytes exposed to B. besnoiti or murine J774A.1 macrophage-like cells in response to E. coli or C. albicans $[23,28]$. Aulik et al. [25] reported that cytochalasin $\mathrm{D}$ reduced the bovine peripheral-blood and alveolar macrophage METosis response to M. haemolytica and its leukotoxin, but, in this same study, cytochalasin D had no significant effect on MET release from THP-1 or RAW 264.7 macrophagelike cells in response to $E$. coli hemolysin. Contrary to this report, Je et al. [26] found that THP-1 macrophage-like cells treated with cytochalasin D were impaired with regard to phagocytosis and MET release in response to $M$. massiliense. These findings have led some authors to speculate that the phagocytosis of particular microorganisms or microbial toxins maybe an important trigger for MET formation [25, 26].

There are also conflicting reports regarding the role of cytoskeletal inhibitors in NET biology. In the original NET description, cytochalasin D effectively prevented phagocytosis of Shigella flexneri or S. aureus but not NET release [5]. Other studies have also reported that cytoskeletal inhibitors had no effect on NET formation in response to Paracoccidioides brasiliensis or gold nanoparticles $[49,50]$. Neeli et al. [51] reported that a $10 \mu \mathrm{M}$ treatment with either the microtubule inhibitor nocodazole or cytochalasin D diminished histone deamination and NET release in response to LPS, and similar results were documented by Jerjomiceva et al. [52] using enrofloxacin-treated bovine neutrophils. The basis for the divergent results of these studies is not clear, but more research is needed to better define the role of the cytoskeleton during the processes of ETosis in neutrophils and macrophages.

A few studies have investigated the role of protein arginine deiminases (PADs) and histone hypercitrullination as an important step in METosis. $\mathrm{PAD}_{4}$-mediated hypercitrullination has been shown to be an important pathway during neutrophil ETosis that leads to chromatin decondensation; inhibition of this pathway impairs NET release [53]. Nakazawa et al. [37] made the observation that $\mathrm{PAD}_{4}$ expression in PMA-differentiated THP-1 cells accompanied $\mathrm{M} 1$ phenotype induction; treatment of these cells with $\mathrm{PAD}_{4}$ siRNA resulted in the inhibition of extracellular DNA release. Mohanan et al. [41] demonstrated that treatment with TNF- $\alpha$ resulted in histone hypercitrullination via $\mathrm{PAD}_{2}$ in RAW 264.7 cells prior to chromatin decondensation and MET release. 
Overall, the limited studies that have evaluated the steps of METosis mirror the findings of neutrophil ETosis, but there are discrepancies in the literature, particularly with regard to the role of ROS and cytoskeleton polymerization during METosis. More work is needed to further define differences in the ROS-dependent and independent pathways of METosis and also whether particular signaling pathways may favor one over the other.

\section{Is METosis a Cell Death Pathway?}

ETosis is considered to be a different cellular pathway from apoptosis as neutrophils undergoing ETosis do not demonstrate typical DNA fragmentation, lack phosphatidylserine localization to the outer leaflet of cellular membrane, and lack typical caspase activation, all hallmarks of cells undergoing apoptosis [6]. ETosis is also notably different from cellular necrosis as, in ETosis, both the nuclear and granular membranes disintegrate, while the plasma membrane is intact [6]. As noted above, reports of alternative forms of ETosis have been documented in neutrophils and eosinophils, and these suggest that not all ETosis pathways end in cell death $[9,45]$.

A few studies have evaluated monocyte and macrophage viability while undergoing METosis. Using fluorescence cell staining for intracellular esterase activity and plasma membrane integrity, Chow et al. [31] revealed that staining of MET-producing RAW 264.7 macrophage-like cells was consistent with a loss of membrane integrity, suggesting that these cells were no longer viable. Vega et al. [39] used a similar approach and noted that mouse J774A.1 macrophage-like cells producing METs were dead within 3 h. Nakazawa et al. [37] used TUNEL staining to evaluate M1-polarized THP-1 cells releasing extracellular DNA after exposure to NETs. The cells demonstrated positive TUNEL staining, which contradicts a report on neutrophils that suggested that NET-producing cells are TUNEL negative [6].

One study evaluated the role of caspase-1 in METosis. Webster et al. [54] illustrated that human peripheralblood monocytes infected with either E. coli or Klebsiella pneumoniae resulted in caspase- 1 activation, which is considered part of some nonapoptotic cell death pathways, particularly pryoptosis. Inhibiting caspase- 1 with the chemical z-YVAD-fmk reduced MET release. With caspase- 1 activation, the authors remarked on a general loss of cell viability over the first $12 \mathrm{~h}$ of infection. Caspase-1, while not previously noted to be activated in neutrophil ETosis, was found to be activated in macrophages in response to NET material and the antibacterial protein LL-37 present on NET fibers [55]. It remains unclear if exposure to MET fibers could induce a similar response in surrounding macrophages, potentially driving further proinflammatory responses including METs.

The sparse data available suggest that METosis is indeed a cell death process, similar to the classic pathways in neutrophils. More work is needed to define the principal triggers and pathways that move a macrophage from other immune responses to METosis. Additional studies are also needed to differentiate the markers of different cellular death pathways, including METosis, and to clarify the conflicting data.

\section{Area of Future Research}

As the interest in METs grows, research will likely focus on differences between macrophages and other cells that produce ETs. In other leukocytes like neutrophils or basophils, macrophage functions can vary dramatically based on differentiation, microenvironment, and polarization states. As such, it may not be surprising that the current literature in this field contains many conflicting reports. Future studies will need to find ways to delineate how subtle shifts in cellular programming, such as macrophage polarization, may direct macrophages to different immune responses against pathogens. Additionally, it has yet to be explored whether METosis occurs globally in all macrophages, including specialized cells like Kupffer cells and microglia. As the NET literature has expanded broadly since the first description in 2004, it seems likely that appreciation of METs and their role in immunity and pathophysiology will continue to gain recognition and stimulate additional studies to add to our current understanding of METosis.

\section{Acknowledgments}

This work was funded by National Institutes of Health grants T32-AI007474 and 2T32HD060554-06A2 (R.S.D.). Additional support was provided by a Career Development Award IK2BX001701 (J.A.G.) from the Office of Medical Research, Department of Veterans Affairs, and by The Global Alliance to Prevent Prematurity and Stillbirth (D.M.A.). Core services including use of the Cell Imaging Shared Resource were performed through support from the Vanderbilt Institute for Clinical and Translational Research program supported by the National Center for Research Resources (grant UL1 RR024975-01) and the National Center for Advancing Translational Sciences (grant 2 UL1 TR000445-06).

\section{Disclosure Statement}

The authors declare no conflicts of interest. 


\section{References}

1 Wynn TA, Chawla A, Pollard JW: Macrophage biology in development, homeostasis and disease. Nature 2013;496:445-455.

2 Gautier EL, Shay T, Miller J, Greter M, Jakubzick C, Ivanov S, Helft J, Chow A, Elpek KG, Gordonov S, Mazloom AR, Ma'ayan A, Chua WJ, Hansen TH, Turley SJ, Merad M, Randolph GJ; Immunological Genome Consortium: Gene-expression profiles and transcriptional regulatory pathways that underlie the identity and diversity of mouse tissue macrophages. Nat Immunol 2012;13:11181128.

3 Murray PJ, Wynn TA: Protective and pathogenic functions of macrophage subsets. Nat Rev Immunol 2011;11:723-737.

4 Ginhoux F, Guilliams M: Tissue-resident macrophage ontogeny and homeostasis. Immunity 2016;44:439-449.

5 Brinkmann V, Reichard U, Goosmann C, Fauler B, Uhlemann Y, Weiss DS, Weinrauch Y, Zychlinsky A: Neutrophil extracellular traps kill bacteria. Science 2004;303:15321535.

6 Fuchs TA, Abed U, Goosmann C, Hurwitz R, Schulze I, Wahn V, Weinrauch Y, Brinkmann V, Zychlinsky A: Novel cell death program leads to neutrophil extracellular traps. J Cell Biol 2007; 176:231-241

7 Wartha F, Henriques-Normark B: ETosis: a novel cell death pathway. Sci Signal 2008; 1:pe25.

8 Möllerherm H, von Köckritz-Blickwede M, Branitzki-Heinemann K: Antimicrobial activity of mast cells: role and relevance of extracellular DNA traps. Front Immunol 2016;7: 265.

9 Yousefi S, Gold JA, Andina N, Lee JJ, Kelly AM, Kozlowski E, Schmid I, Straumann A, Reichenbach J, Gleich GJ, Simon HU: Catapult-like release of mitochondrial DNA by eosinophils contributes to antibacterial defense. Nat Med 2008;14:949-953.

10 Schorn C, Janko C, Latzko M, Chaurio R, Schett G, Herrmann M: Monosodium urate crystals induce extracellular DNA traps in neutrophils, eosinophils, and basophils but not in mononuclear cells. Front Immunol 2012;3:277.

11 Morshed M, Hlushchuk R, Simon D, Walls AF, Obata-Ninomiya K, Karasuyama $H$, Djonov V, Eggel A, Kaufmann T, Simon HU, Yousefi S: NADPH oxidase-independent formation of extracellular DNA traps by basophils. J Immunol 2014;192:5314-5323.

12 von Köckritz-Blickwede $\mathrm{M}$, Goldmann O, Thulin P, Heinemann K, Norrby-Teglund A, Rohde M, Medina E: Phagocytosis-independent antimicrobial activity of mast cells by means of extracellular trap formation. Blood 2008;111:3070-3080.
13 Schorn C, Janko C, Krenn V, Zhao Y, Muñoz LE, Schett G, Herrmann M: Bonding the foe: NETting neutrophils immobilize the proinflammatory monosodium urate crystals. Front Immunol 2012;3:376.

14 Pang L, Hayes CP, Buac K, Yoo DG, Rada B: Pseudogout-associated inflammatory calcium pyrophosphate dihydrate microcrystals induce formation of neutrophil extracellular traps. J Immunol 2013;190:6488-6500.

15 Gupta AK, Hasler P, Holzgreve W, Gebhardt S, Hahn S: Induction of neutrophil extracellular DNA lattices by placental microparticles and IL-8 and their presence in preeclampsia. Hum Immunol 2005;66:1146-1154.

16 Fuchs TA, Brill A, Duerschmied D, Schatzberg D, Monestier M, Myers DD Jr, Wrobleski SK, Wakefield TW, Hartwig JH, Wagner DD: Extracellular DNA traps promote thrombosis. Proc Natl Acad Sci USA 2010;107: 15880-15885.

17 Simon D, Simon HU, Yousefi S: Extracellular DNA traps in allergic, infectious, and autoimmune diseases. Allergy 2013;68:409-416.

18 Söderberg D, Segelmark M: Neutrophil extracellular traps in ANCA-associated vasculitis. Front Immunol 2016;7:256.

19 Delgado-Rizo V, Martínez-Guzmán MA, Iñiguez-Gutierrez L, García-Orozco A, Alvarado-Navarro A, Fafutis-Morris M: Neutrophil extracellular traps and its implications in inflammation: an overview. Front Immunol 2017;8:81.

20 Goldmann O, Medina E: The expanding world of extracellular traps: not only neutrophils but much more. Front Immunol 2012;3: 420.

21 Arksey H, O’Malley L: Scoping studies: towards a methodological framework. Int J Soc Res Methodol 2005;8:19-32.

22 Halder LD, Abdelfatah MA, Jo EA, Jacobsen ID, Westermann M, Beyersdorf N, Lorkowski S, Zipfel PF, Skerka C: Factor H binds to extracellular DNA traps released from human blood monocytes in response to Candida albicans. Front Immunol 2016;7:671.

23 Muñoz-Caro T, Silva LM, Ritter C, Taubert A, Hermosilla C: Besnoitia besnoiti tachyzoites induce monocyte extracellular trap formation. Parasitol Res 2014;113:4189-4197.

24 Hellenbrand KM, Forsythe KM, Rivera-Rivas JJ, Czuprynski CJ, Aulik NA: Histophilus somni causes extracellular trap formation by bovine neutrophils and macrophages. Microb Pathog 2013;54:67-75.

25 Aulik NA, Hellenbrand KM, Czuprynski CJ: Mannheimia haemolytica and its leukotoxin cause macrophage extracellular trap formation by bovine macrophages. Infect Immun 2012;80:1923-1933.

26 Je S, Quan H, Yoon Y, Na Y, Kim BJ, Seok SH: Mycobacterium massiliense induces macrophage extracellular traps with facilitating bacterial growth. PLoS One 2016;11:e0155685.
27 O’Sullivan KM, Lo CY, Summers SA, Elgass KD, McMillan PJ, Longano A, Ford SL, Gan PY, Kerr PG, Kitching AR, Holdsworth SR: Renal participation of myeloperoxidase in antineutrophil cytoplasmic antibody (ANCA)associated glomerulonephritis. Kidney Int 2015;88:1030-1046.

28 Liu P, Wu X, Liao C, Liu X, Du J, Shi H, Wang X, Bai X, Peng P, Yu L, Wang F, Zhao Y, Liu M: Escherichia coli and Candida albicans induced macrophage extracellular trap-like structures with limited microbicidal activity. PLoS One 2014;9:e90042.

29 Pérez D, Muñoz MC, Molina JM, MuñozCaro T, Silva LM, Taubert A, Hermosilla C, Ruiz A: Eimeria ninakohlyakimovae induces NADPH oxidase-dependent monocyte extracellular trap formation and upregulates IL-12 and TNF- $\alpha$, IL- 6 and CCL2 gene transcription. Vet Parasitol 2016;227:143-150.

30 Wong KW, Jacobs WR Jr: Mycobacterium tuberculosis exploits human interferon gamma to stimulate macrophage extracellular trap formation and necrosis. J Infect Dis 2013;208: 109-119.

31 Chow OA, von Köckritz-Blickwede M, Bright AT, Hensler ME, Zinkernagel AS, Cogen AL, Gallo RL, Monestier M, Wang Y, Glass CK, Nizet V: Statins enhance formation of phagocyte extracellular traps. Cell Host Microbe 2010;8:445-454.

32 Bonne-Année S, Kerepesi LA, Hess JA, Wesolowski J, Paumet F, Lok JB, Nolan TJ, Abraham D: Extracellular traps are associated with human and mouse neutrophil and macrophage mediated killing of larval Strongyloides stercoralis. Microb Infect 2014;16:502-511.

33 Yang F, Feng C, Zhang X, Lu J, Zhao Y: The diverse biological functions of neutrophils, beyond the defense against infections. Inflammation 2017;40:311-323.

34 Andzinski L, Kasnitz N, Stahnke S, Wu CF, Gereke M, von Köckritz-Blickwede M, Schilling B, Brandau S, Weiss S, Jablonska J: Type I IFNs induce anti-tumor polarization of tumor associated neutrophils in mice and human. Int J Cancer 2016;138:1982-1993.

35 Shrestha S, Kim SY, Yun YJ, Kim JK, Lee JM, Shin M, Song DK, Hong CW: Retinoic acid induces hypersegmentation and enhances cytotoxicity of neutrophils against cancer cells. Immunol Lett 2017;182:24-29.

36 Villanueva E, Yalavarthi S, Berthier CC, Hodgin JB, Khandpur R, Lin AM, Rubin CJ, Zhao W, Olsen SH, Klinker M, Shealy D, Denny MF, Plumas J, Chaperot L, Kretzler M, Bruce AT, Kaplan MJ: Netting neutrophils induce endothelial damage, infiltrate tissues, and expose immunostimulatory molecules in systemic lupus erythematosus. J Immunol 2011; 187:538-552. 
37 Nakazawa D, Shida H, Kusunoki Y, Miyoshi A, Nishio S, Tomaru U, Atsumi T, Ishizu A: The responses of macrophages in interaction with neutrophils that undergo NETosis. J Autoimmun 2016;67:19-28.

38 Bryukhin GV, Shopova AV: Characteristics of mononuclear extracellular traps in the offspring of female rats with drug-induced hepatitis. Bull Exp Biol Med 2015;159:435-437.

39 Vega VL, Crotty Alexander LE, Charles W, Hwang JH, Nizet V, De Maio A: Activation of the stress response in macrophages alters the M1/M2 balance by enhancing bacterial killing and IL-10 expression. J Mol Med (Berl) 2014; 92:1305-1317.

40 Shen F, Tang X, Cheng W, Wang Y, Wang C, Shi X, An Y, Zhang Q, Liu M, Liu B, Yu L: Fosfomycin enhances phagocyte-mediated killing of Staphylococcus aureus by extracellular traps and reactive oxygen species. Sci Rep 2016;6:19262.

41 Mohanan S, Horibata S, McElwee JL, Dannenberg AJ, Coonrod SA: Identification of macrophage extracellular trap-like structures in mammary gland adipose tissue: a preliminary study. Front Immunol 2013;4:67.

42 Stoiber W, Obermayer A, Steinbacher P, Krautgartner WD: The role of reactive oxygen species (ROS) in the formation of extracellular traps (ETs) in humans. Biomolecules 2015; 5:702-723.

43 Papayannopoulos V, Metzler KD, Hakkim A, Zychlinsky A: Neutrophil elastase and myeloperoxidase regulate the formation of neutrophil extracellular traps. J Cell Biol 2010;191: 677-691.

44 Remijsen Q, Kuijpers TW, Wirawan E, Lippens $S$, Vandenabeele $\mathrm{P}$, Vanden Berghe $\mathrm{T}$ : Dying for a cause: NETosis, mechanisms behind an antimicrobial cell death modality. Cell Death Differ 2011;18:581-588.

45 Yousefi S, Mihalache C, Kozlowski E, Schmid I, Simon HU: Viable neutrophils release mitochondrial DNA to form neutrophil extracellular traps. Cell Death Differ 2009;16 1438-1444.
46 Pilsczek FH, Salina D, Poon KK, Fahey C, Yipp BG, Sibley CD, Robbins SM, Green FH, Surette MG, Sugai M, Bowden MG, Hussain M, Zhang K, Kubes P: A novel mechanism of rapid nuclear neutrophil extracellular trap formation in response to Staphylococcus aureus. J Immunol 2010;185:7413-7425.

47 King PT, Sharma R, O'Sullivan K, Selemidis S, Lim S, Radhakrishna N, Lo C, Prasad J, Callaghan J, McLaughlin P, Farmer M, Steinfort D, Jennings B, Ngui J, Broughton BR, Thomas B, Essilfie AT, Hickey M, Holmes PW, Hansbro P, Bardin PG, Holdsworth SR: Nontypeable Haemophilus influenzae induces sustained lung oxidative stress and protease expression. PLoS One 2015;10:e120371.

48 Metzler KD, Fuchs TA, Nauseef WM, Reumaux D, Roesler J, Schulze I, Wahn V, Papayannopoulos V, Zychlinsky A: Myeloperoxidase is required for neutrophil extracellular trap formation: implications for innate immunity. Blood 2011;117:953-959.

49 Bartneck M, Keul HA, Zwadlo-Klarwasser G, Groll J: Phagocytosis independent extracellular nanoparticle clearance by human immune cells. Nano Lett 2010;10:59-63.

50 Mejia SP, Cano LE, López JA, Hernandez O, González A: Human neutrophils produce extracellular traps against Paracoccidioides brasiliensis. Microbiology 2015;161:10081017.

51 Neeli I, Dwivedi N, Khan S, Radic M: Regulation of extracellular chromatin release from neutrophils. J Innate Immun 2009;1:194-201.

52 Jerjomiceva N, Seri H, Völlger L, Wang Y, Zeitouni N, Naim HY, von Köckritz-Blickwede M: Enrofloxacin enhances the formation of neutrophil extracellular traps in bovine granulocytes. J Innate Immun 2014;6: 706-712.
53 Wang Y, Li M, Stadler S, Correll S, Li P, Wang D, Hayama R, Leonelli L, Han H, Grigoryey SA, Allis CD, Coonrod SA: Histone hypercitrullination mediates chromatin decondensation and neutrophil extracellular trap formation. J Cell Biol 2009;184:205-213.

54 Webster SJ, Daigneault M, Bewley MA, Preston JA, Marriott HM, Walmsley SR, Read RC, Whyte MK, Dockrell DH: Distinct cell death programs in monocytes regulate innate responses following challenge with common causes of invasive bacterial disease. J Immunol 2010;185:2968-2979.

55 Kahlenberg JM, Carmona-Rivera C, Smith CK, Kaplan MJ: Neutrophil extracellular trap-associated protein activation of the NLRP3 inflammasome is enhanced in lupus macrophages. J Immunol 2013;190:12171226.

56 Reichel M, Muñoz-Caro T, Sanchez Contreras G, Rubio García A, Magdowski G, Gärtner U, Taubert A, Hermosilla C: Harbour seal (Phoca vitulina) PMN and monocytes release extracellular traps to capture the apicomplexan parasite Toxoplasma gondii. Dev Comp Immunol 2015;50:106-115.

57 Jönsson BE, Bylund J, Johansson BR, Telemo E, Wold AE: Cord-forming mycobacteria induce DNA meshwork formation by human peripheral blood mononuclear cells. Pathog Dis 2013;67:54-66.

58 Pijanowski L, Scheer M, Verburg-van Kemenade BM, Chadzinska M: Production of inflammatory mediators and extracellular traps by carp macrophages and neutrophils in response to lipopolysaccharide and/or interferon- $\gamma 2$. Fish Shellfish Immunol 2015; 42:473-482.

59 Korir ML, Laut C, Rogers LM, Plemmons JA, Aronoff DM, Manning SD: Differing mechanisms of surviving phagosomal stress among group B Streptococcus strains of varying genotypes. Virulence 2016, Epub ahead of print. 